\title{
Gureger Urinary biomarkers of renal transplant outcome
}

\author{
Julie Ho ${ }^{\mathrm{a}, \mathrm{b}, \mathrm{c}}$, David N. Rush ${ }^{\mathrm{a}}$, and Peter W. Nickerson ${ }^{\mathrm{a}, \mathrm{b}, \mathrm{c}}$
}

\begin{abstract}
Purpose of review
Renal allograft loss remains an important cause of morbidity and mortality. The objective of this review was to provide a rationale for noninvasive monitoring to identify patients at high risk for graft loss; discuss key steps in prognostic biomarker development from bench-to-bedside; and review promising biomarkers for late renal allograft outcomes.
\end{abstract}

\section{Recent findings}

In a multicentre prospective cohort, early 6-month urinary CCL2 was demonstrated to be associated with the development of 24-month interstitial fibrosis/tubular atrophy and inflammation (IFTA+i). These findings were extended to a single centre cohort, which showed that 6-month urinary CCL2 was a predictor of death-censored graft loss independent of donor-specific antibody and delayed graft function. In a large, multicentre prospective observational study (CTOT-01), 6-month urinary CXCL9 was significantly associated with more than $30 \%$ decline of graft function at 24 months.

\section{Summary \\ Urinary chemokines may identify recipients who are at high risk of graft loss. The early detection of high- risk recipients may allow for more intensive posttransplant surveillance; avoidance of drug minimization/ withdrawal protocols; and the identification of patients who may benefit from enrolment in novel interventional trials. Prospective trials are needed to demonstrate that urinary chemokine-guided posttransplant surveillance strategies improve long-term graft outcomes. \\ Keywords \\ CCL2, CXCL10, CXCL9, noninvasive monitoring, posttransplant surveillance, urine microRNA}

\section{INTRODUCTION}

Transplantation is the therapy of choice for many patients with end-stage renal disease as it enhances both survival and quality of life. Graft loss, however, remains a clinically significant problem. Indeed, the return to dialysis following graft loss is associated with a three-fold increased risk of death, immunological sensitization that may impede retransplantation, a lower quality of life, and increased costs [1,2]. United States Renal Data System (USRDS) studies demonstrate that adjusted patient survival after graft loss is less than $40 \%$ at 10 years compared with greater than a 75\% 10-year survival with a functioning renal transplant [2]. Similarly, Canadian Organ Replacement Registry (CORR) data also demonstrate that graft loss is an independent predictor of mortality, with a three-fold increased risk of death compared with patients who maintain graft function [1].

Important gains have been made in overall graft survival. From 1991 to 2007, renal allograft half-life has improved from 10.1 and 15.8 years, to 14.7 and 26.6 years for the recipients of deceased and living donor allografts, respectively [3], and these gains have remained stable [4]. These observations are supported by improved long-term renal histology on protocol biopsies at both 1 and 5 years [5]. Although the majority of these gains is attributable to improved early outcomes with modern immunosuppression, there remains a small but appreciable improvement in late death-censored graft loss [6]. Nevertheless, improvement of long-term outcomes remains a major hurdle for the transplantation community.

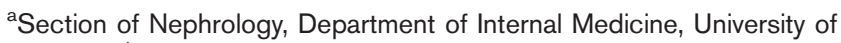
Manitoba, ${ }^{\mathrm{b}}$ Manitoba Centre for Proteomics and Systems Biology, Health Sciences Centre and 'Department of Immunology, University of Manitoba, Winnipeg, Manitoba, Canada

Correspondence to Julie Ho, MD FRCPC, Associate Professor, Internal Medicine and Immunology, Sections of Nephrology and Biomedical Proteomics, GE421C Health Sciences Centre, 820 Sherbrook Street, Winnipeg, Manitoba R3A 1R9, Canada. Tel: +1 204787 3115; fax: +1 204787 3326; e-mail: jho@hsc.mb.ca
}

Curr Opin Organ Transplant 2015, 20:476-481

DOI:10.1097/MOT.0000000000000208 


\section{KEY POINTS}

- Early, noninvasive prognostic markers would be useful to identify patients at high risk of graft functional decline and graft loss.

- Six-month urinary CCL2 predicts the development of IFTA and inflammation (IFTA+i) at 24 months and death-censored graft loss in independent cohorts.

- Six-month urinary CXCL9 predicts the decline in eGFR more than $30 \%$ from 6 to 24 months in a multicentre setting.

- Urinary chemokines are readily measured by ELISA.

- Prospective studies are required to demonstrate that urinary chemokine-guided monitoring strategies improve long-term graft outcomes.

\section{ARE THE CAUSES OF LATE GRAFT LOSS PREVENTABLE?}

Data demonstrate that the underlying causes of graft failure are largely identifiable $\left[7,8^{\mathbf{*}}\right]$ and primarily immune-mediated [9-12]. In a protocol biopsy series, El-Zoghby et al. [7] reported that death-censored graft loss was attributable to glomerular diseases (36.6\%, including transplant glomerulopathy); fibrosis/atrophy (30.7\%); medical/surgical conditions (16.3\%); acute rejection (11.8\%); and unclassifiable (4.6\%). These findings are consistent with Naesens et al. [8"] who recently observed that specific causes of graft loss were present in $69.4 \%$ of indication biopsies, with T-cell-mediated rejection (TCMR), antibody-mediated rejection, and transplant glomerulopathy being the dominant features of $52.8 \%$ of indication biopsies prior to graft loss. These observations give reason for hope, as immunemediated causes of graft loss may be treatable if identified early.

What of the third of biopsies with significant histological chronicity identified in the absence of a specific diagnosis $\left[7,8^{-}\right]$? Clearly, the causes for these findings may be multifactorial in nature and not necessarily immune-related, such as donor age and quality. Efforts to characterize chronic, 'nonspecific' histological lesions may, however, ultimately contribute to improving long-term graft outcomes [13]. The Banff criteria are a working classification in evolution, and alloimmune inflammation may exist that is currently below the threshold to be diagnosed as rejection, despite having a significant negative impact on graft survival [14]. Indeed, the successes of modern immunosuppression may have left a legacy of subtler forms of alloimmune inflammation that are currently underrecognized.
Multiple groups have now demonstrated that interstitial fibrosis/tubular atrophy + inflammation $($ IFTA $+\mathrm{i})$ is a significant prognostic marker for graft loss $[13,15-20]$. Even mild degrees of IFTA $+\mathrm{i}$ in which the degree of inflammation does not meet diagnostic criteria for Banff borderline rejection are strongly and independently associated with renal allograft functional decline and death-censored graft loss $[15,16,18]$. It is noteworthy that IFTA $+i$ is associated with episodes of acute rejection and increased levels of HLA mismatch $[15,17]$, as well as a rejectionlike gene expression signature $[16,20]$. Taken together, these findings expand upon earlier work that demonstrates that subclinical TCMR is associated with poor graft outcomes [21-24] and suggests that IFTA $+\mathrm{i}$ may represent an ongoing, low-grade cellular rejection state that is not recognized in the current Banff schema $[15,25]$ and may be amenable to intervention.

\section{REVIEW}

\section{What is the role for urinary biomarkers?}

Urinary biomarkers could play a key role in improving long-term graft survival. Noninvasive monitoring strategies have the potential to identify early alloimmune inflammation when the injury may still be reversible. Indeed, the early identification of such high-risk recipients may call for more intensive posttransplant surveillance, avoidance of drug minimization/withdrawal protocols, and permit their enrolment in novel interventional trials, such as increased immunosuppression for IFTA $+\mathrm{i}$.

\section{Prognostic biomarker development: key steps from bench to bedside}

Biomarker development has been broadly categorized into the following stages: discovery, performance evaluation, and impact determination [26]. Ideally, a prognostic marker would be as follows:

\section{Discovery}

(1) An early marker for late graft outcomes, not a concomitant marker for established, irreversible pathologies.

(2) Predict appropriate surrogate outcomes for graft loss (e.g. histology, graft function).

(3) Possess sufficient power, especially if multiple biomarkers are being assessed.

(4) Highly sensitive and specific.

\section{Performance evaluation}

(1) Correlate with hard, clinically relevant outcomes - graft loss. 
(2) Validated independently in separate, preferably multicentre, cohorts to demonstrate reproducibility and generalizability.

(3) Evaluated in unselected patient cohorts to characterize population-based diagnostic performance.

(4) Have the capacity for high-throughput, inexpensive, robust and reproducible assays, with accessible laboratory equipment that would be available in clinical laboratories.

(5) Follow Good Laboratory Practice Guidelines.

\section{Impact determination}

(1) Exceed clinical prediction models for graft loss (e.g. serum creatinine, proteinuria).

(2) Demonstrate improvement of long-term allograft outcomes in a prospective interventional trial.

Many studies have sought to identify novel, noninvasive urinary biomarkers for concomitant IFTA or graft dysfunction. These discovery studies have used many different approaches, including Luminex multiplex assays, proteomics, and peptidomics [27-31]. Although these are helpful for determining novel targets and mapping the underlying pathophysiological pathways activated in IFTA, this review will focus on biomarkers measured early in the posttransplant course that correlates with late outcomes.

There are a number of limitations inherent to evaluating early prognostic markers for death-censored graft loss. Specifically, renal allograft half-lives range between 15.8 and 26.6 years [3]; therefore, the majority of prospective studies are forced to rely on surrogate outcomes. Conversely, retrospective studies with sufficient follow-up to evaluate graft loss may be confounded by an era effect with immunosuppression. This lead time also highlights the difficulties in performing prospective, interventional trials to demonstrate the utility of a prognostic biomarker for improving graft outcomes. With these limitations in mind, we will discuss promising, prognostic urinary biomarkers for late graft outcomes.

\section{EARLY URINARY BIOMARKERS FOR LATE ALLOGRAFT OUTCOMES}

\section{Urinary-cell microRNA}

MicroRNAs (miRNAs) are small, noncoding RNAs that regulate gene expression through posttranscriptional repression of their target RNAs [32]. In a series of nicely designed discovery microarray experiments, Maluf et al. [33] identified differentially expressed urinary and renal miRNAs [34] in several highly selected, cross-sectional, and longitudinal cohorts, and selected targets were confirmed with RT-QPCR. Twelve miRNA candidates were identified for evaluation in a prospective, longitudinal cohort $(n=66)$ with early 3-6-month urines and 24-month outcomes [33]. Early posttransplant urines in patients who developed 24-month poor graft function (eGFR less than $40 \mathrm{ml} / \mathrm{min}$ ) and IFTA on last protocol biopsy ( $\mathrm{ci} \geq 1 \mathrm{ct} \geq 1$ ) versus stable function/normal histology showed that miR-99a was differentially expressed at 3 months in patients who developed poor graft function at 24 months $(P=0.03)$, whereas miR-140-3p, miR-200*, and miR$200 \mathrm{~b}$ were only borderline significant.

Although these data are informative from a pathophysiological perspective, there are some limitations. First, the clinical characteristics of the longitudinal evaluation group were not reported separately from the stable function/normal histology versus poor graft function/IFTA groups, so it is unknown whether renal function or proteinuria were already different at 3-6 months posttransplant. Therefore, it is not possible to determine whether urinary miRNAs contribute additional prognostic information for graft functional decline/IFTA beyond routine clinical measures. They did demonstrate that concomitant proteinuria and urinary miRNAs were not correlated at the early time point, which suggests that urinary miRNAs may add additional information, but this was not further characterized. Finally, they evaluated IFTA as a histological surrogate of poor graft outcomes, instead of IFTA $+\mathrm{i}$ that may provide better prognostic information. Therefore, these findings need to be evaluated and validated in an independent cohort.

\section{Urinary proteins}

Nauta et al. [35] evaluated multiple potential urinary markers as predictors of long-term renal allograft outcomes including albuminuria, proteinuria, neutrophil gelatinase-associated lipocalin (NGAL), heart fatty-acid binding protein, kidney injury molecule-1 (KIM- 1 ), and $N$-acetyl- $\beta$-D-glucosaminidase. Prevalent patients were enrolled at a median of 5.9 years posttransplant and albuminuria was found to be an independent predictor of subsequent graft dysfunction and loss, and interestingly outperformed total proteinuria [35]. This study did not account for pretransplant or de-novo donor-specific antibody (DSA) and histology was not available to correlate with outcomes. Szeto et al. [36] evaluated urinary KIM-1, NGAL, and IL-18 expression in a cross-sectional cohort of prevalent renal transplant 
patients and reported that urine KIM-1 mRNA was a multivariate predictor for graft loss. These findings have yet to be independently confirmed and KIM-1 protein was not associated with death-censored graft loss in Nauta's study after adjusting for albuminuria [35]. These prevalent studies $[35,36]$ may also be further confounded by survivor bias.

In a prospective, multicentre observational study of incident deceased donor renal transplant recipients, Hall et al. [37] showed that urinary NGAL and interleukin-18 (IL-18) within the first postoperative day were associated with composite of poor allograft function at 1 year (eGFR $<30 \mathrm{ml} / \mathrm{min}$ ) and return to dialysis. The outcome group had, however, a higher incidence of delayed graft function (DGF) $(P=0.03)$ and when DGF was added to the prediction models, both the net reclassification and integrated discrimination index indices dropped $5 \%$ or less (not significant) for NGAL and IL-18, suggesting that the biomarkers may not contribute significant additional information to the base clinical model [37].

In a single centre, prospective observational study, Amer et al. [38] evaluated the prognostic value of 1-year posttransplant urinary total protein, albumin, retinol binding protein, $\alpha 1$-microglobulin, IgG, and IgM on death-censored graft survival. These urinary proteins did not distinguish underlying 1-year protocol biopsy histology. Urinary retinol binding protein was found to be an independent predictor for death-censored graft loss $(n=12$, HR $1.52, P=0.001)$. The diagnostic characteristics [area under the curve receiver operating characteristic (AUC)] for graft loss were, however, not reported; therefore, these findings need to be independently validated [38].

\section{URINARY CHEMOKINES}

\section{Urinary CCL2}

CCL2 is a CCR2 receptor chemokine produced by renal tubular and glomerular epithelial cells, and infiltrating leukocytes. CCL2 is chemoattractant for monocytes/macrophages, T-lymphocytes, and natural killer cells $[39,40]$, and may contribute to generating memory $\mathrm{CD}^{+}{ }^{+} \mathrm{T}$-lymphocytes [41]. In a prospective, multicentre study of adult renal transplant recipients with serial protocol biopsies and urine samples, we evaluated early predictors for 24-month histological outcomes [42]. Interestingly, 6-month urinary CCL2:Cr was associated with the development of worsening allograft function and IFTA at 24 months, and indeed was an independent predictor of 24-month IFTA [odds ratio (OR) 1.049, $P=0.024]$ after adjusting for donor age, DGF, deceased donation, and ACEi/ARB exposure [42]. Emerging data, however, indicate that IFTA alone is not prognostically significant, whereas IFTA $+\mathrm{i}$ is an important predictor for graft loss [13,15-20]. Therefore, we confirmed that 6-month urinary CCL2: $\mathrm{Cr}$ was an independent predictor for the development of 24-month IFTA+i (OR 1.88, AUC $0.641, P=0.01)$ in this cohort of patients [43"'].

Even though IFTA $+\mathrm{i}$ is a more appropriate surrogate for graft loss than IFTA alone, it still remains a surrogate. Therefore, in a single centre, prospective observational cohort, we evaluated the association between 6-month urinary CCL2:Cr and graft loss [44]. Notably, we confirmed that CCL2 is an independent predictor for death-censored graft loss (HR 2.20, $P=0.01$ ) after adjustment for pretransplant de-novo DSA and DGF, and outperformed renal function and proteinuria. Furthermore, in a multivariate model using variables known to a clinician at 6 months posttransplant (CCL2, recipient age, and DGF) yielded an AUC 0.87 for predicting deathcensored graft loss with a positive predictive value 0.96 [44]. These findings need to be validated in an independent patient population with follow-up for graft loss.

\section{Urinary CXCL9}

CXCR3 is a chemokine receptor that is expressed by activated $\mathrm{T}$ cells and natural killer cells and binds CXCL9, CXCL10, and CXCL11 [45]. CXCL9 and CXCL10 are secreted by infiltrating inflammatory cells, renal tubular, and mesangial cells, and recruit leukocytes to mediate the Th1 response $[46,47]$. In a prospective, multicentre observational study of adult and pediatric renal transplant recipients, the CTOT-01 group evaluated multiple biomarkers, and validated urinary CXCL9 as a noninvasive marker for biopsy-proven acute rejection with a negative predictive value 0.92 [48]. Interestingly, they found that 6-month urinary CXCL9 levels were associated with the risk of subsequent $6-24$-month acute rejection (OR 4.695, AUC 0.88, $P=0.005$ ). Furthermore, they established that 6-month urinary CXCL9 predicted a decline in eGFR more than 30\% between 6 and 24 months (AUC 0.68, $P=0.001$, NPV 0.925) [48].

This work builds on previous data that demonstrate urinary CXCR3 chemokines (CXCL9, CXCL10) are sensitive, noninvasive markers of subclinical and clinical TCMR [27,49-59] and validates it in a large, unselected multicentre cohort [48]. Notably, in a prospective, observational single centre study Matz et al. described that elevated urinary CXCL10 within the first month posttransplant was associated with GFR $<45 \mathrm{ml} / \mathrm{min}$ at 
6 months (AUC 0.68). The CTOT-01 study extends these findings to urinary CXCL9 in a multicentre study setting with longer follow-up.

\section{CONCLUSION}

Taken together, these data suggest that urinary chemokines, CCL2 and CXCL9/10, are significantly associated with the development of worsening histology, graft functional decline, and death-censored graft loss. Critically, CCL2 is independent of DSA suggesting that it predicts alternate pathways to graft loss from antibody-mediated rejection. These findings need to be independently validated to determine if the early interaction of CCL2, CXCL9/10, and DSA combines to provide an overall stronger prediction model for graft loss.

Interestingly, urinary chemokines measured at 6 months have been remarkably consistent for predicting late graft outcomes. We speculate that this may be due to a combination of factors. The earlier time points may be confounded with noise due to ischaemia-reperfusion injury and early acute rejection. Furthermore, immunosuppression minimization or withdrawal frequently reaches its nadir at 6 months posttransplant. Low-grade alloimmune inflammation that persists in the setting of stable, reduced immunosuppression may be most predictive for long-term outcomes, so the recipient immune profile at this time may be most predictive, and be characterized in part by elevated urinary chemokine levels.

Finally, urinary CCL2, CXCL9/10 are readily measured by ELISA, which is a straightforward assay for translating from bench to bedside. Ultimately, prospective interventional trials are required to demonstrate that posttransplant monitoring strategies utilizing urinary chemokine monitoring improve long-term graft outcomes.

\section{Acknowledgements}

The authors would like to thank Evelyn Roloff and Rhonda Stevenson for their assistance with preparation of the manuscript.

\section{Financial support and sponsorship}

This work was funded by the Canadian Institutes of Health Research.

This manuscript was made possible by operating support from the Canadian Institutes of Health Research [CIHR grant 287559 (J.H.), CIHR grant 310533 (P.N.)]. J.H. holds salary support from the Manitoba Medical Services Foundation Dr FW Du Val Clinical Research Professorship and P.N. is supported by the Flynn Family Chair in Renal Transplantation, University of Manitoba.

\section{Conflicts of interest}

There are no conflicts of interest.

\section{REFERENCES AND RECOMMENDED}

\section{READING}

Papers of particular interest, published within the annual period of review, have been highlighted as:

- of special interest

- of outstanding interest

1. Knoll G, Muirhead N, Trpeski L, et al. Patient survival following renal transplant failure in Canada. Am J Transplant 2005; 5:1719-1724.

2. Kaplan B, Meier-Kriesche H-U. Death after graft loss: an important late study endpoint in kidney transplantation. Am J Transplant 2002; 2:970-974.

3. OPTN/SRTR 2010 Annual Data Report. Dept. of Health \& Human Services, Health Resources \& Services Administration, Healthcare Systems Bureau, Division of Transplantation. Am J Transplant 2012; 12 (Suppl 1):22-23.

4. Matas AJ, Smith JM, Skeans MA, et al. OPTN/SRTR 2013 Annual Data Report: kidney. Am J Transplant 2015; 15 (Suppl 2):1-34.

5. Stegall MD, Park WD, Larson TS, et al. The histology of solitary renal allografts at 1 and 5 years after transplantation. Am J Transplant 2011; 11:698-707.

6. Lamb KE, Lodhi S, Meier-Kriesche H-U. Long-term renal allograft survival in the United States: a critical reappraisal. Am J Transplant 2011; 11:450-462.

7. El-Zoghby ZM, Stegall MD, Lager DJ, et al. Identifying specific causes of kidney allograft loss. Am J Transplant 2009; 9:527-535.

8. Naesens M, Kuypers DRJ, De Vusser K, et al. The histology of kidney

- transplant failure: a long-term follow-up study. Transplantation 2014; 98: $427-435$.

This detailed single-centre analysis with indication biopsies demonstrated the specific causes of renal allograft loss, with additional granularity on coexisting pathologies.

9. Wiebe C, Gibson IW, Blydt-Hansen TD, et al. Evolution and clinical pathologic correlations of de novo donor-specific HLA antibody post kidney transplant. Am J Transplant 2012; 12:1157-1167.

10. Gourishankar S, Leduc R, Connett J, et al. Pathological and clinical characterization of the 'troubled' transplant: data from the DeKAF study. Am J Transplant 2010; 10:324-330.

11. Einecke G, Sis B, Reeve J, et al. Antibody-mediated microcirculation injury is the major cause of late kidney transplant failure. Am J Transplant 2009; 9:2520-2531.

12. Sellares J, de Freitas DG, Mengel $M$, et al. Understanding the causes of kidney transplant failure: the dominant role of antibody-mediated rejection and nonadherence. Am J Transplant 2012; 12:388-399.

13. Matas AJ, Leduc $R$, Rush $D$, et al. Histopathologic clusters differentiate subgroups within the nonspecific diagnoses of CAN or CR: preliminary data from the DeKAF study. Am J Transplant 2010; 10:315-323.

14. Toki D, Zhang W, Hor KKLM, et al. The role of macrophages in the development of human renal allograft fibrosis in the first year after transplantation. Am J Transplant 2014; 14:2126-2136.

15. Cosio FG, Grande JP, Wadei H, et al. Predicting subsequent decline in kidney allograft function from early surveillance biopsies. Am J Transplant 2005; 5:2464-2472.

16. Park WD, Griffin MD, Cornell LD, et al. Fibrosis with inflammation at one year predicts transplant functional decline. J Am Soc Nephrol 2010; 21:19871997.

17. Gago M, Cornell LD, Kremers WK, et al. Kidney allograft inflammation and fibrosis, causes and consequences. Am J Transplant 2012; 12:1199-1207.

18. Mannon RB, Matas AJ, Grande J, et al. Inflammation in areas of tubular atrophy in kidney allograft biopsies: a potent predictor of allograft failure. Am J Transplant 2010; 10:2066-2073.

19. Shishido $\mathrm{S}$, Asanuma $\mathrm{H}, \mathrm{Nakai} \mathrm{H}$, et al. The impact of repeated subclinical acute rejection on the progression of chronic allograft nephropathy. J Am Soc Nephrol 2003; 14:1046-1052.

20. Mengel $M$, Reeve J, Bunnag $S$, et al. Scoring total inflammation is superior to the current Banff inflammation score in predicting outcome and the degree of molecular disturbance in renal allografts. Am J Transplant 2009; 9:18591867.

21. Moreso $F$, Ibernon $M$, Gomà $M$, et al. Subclinical rejection associated with chronic allograft nephropathy in protocol biopsies as a risk factor for late graft loss. Am J Transplant 2006; 6:747-752.

22. Kurtkoti J, Sakhuja V, Sud K, et al. The utility of 1- and 3-month protocol biopsies on renal allograft function: a randomized controlled study. Am J Transplant 2008; 8:317-323.

23. Rush D, Nickerson $P$, Gough J, et al. Beneficial effects of treatment of early subclinical rejection: a randomized study. J Am Soc Nephrol 1998; 9:21292134.

24. Loupy A, Vernerey D, Tinel C, et al. Subclinical rejection phenotypes at 1 year posttransplant and outcome of kidney allografts. J Am Soc Nephrol 2015; Epub ahead of print. doi:10.1681/ASN.2014040399. 
25. Haas M, Sis B, Racusen LC, et al. Banff 2013 meeting report: inclusion of c4d-negative antibody-mediated rejection and antibody-associated arterial lesions. Am J Transplant 2014; 14:272-283.

26. Parikh $\mathrm{CR}$, Thiessen-Philbrook $\mathrm{H}$. Key concepts and limitations of statistical methods for evaluating biomarkers of kidney disease. J Am Soc Nephrol 2014; 25:1621-1629.

27. $\mathrm{Hu} \mathrm{H}, \mathrm{Kwun} \mathrm{J}$, Aizenstein BD, Knechtle SJ. Noninvasive detection of acute and chronic injuries in human renal transplant by elevation of multiple cytokines/ chemokines in urine. Transplantation 2009; 87:1814-1820.

28. Anglicheau $D$, Muthukumar $T$, Hummel $A$, et al. Discovery and validation of a molecular signature for the noninvasive diagnosis of human renal allograft fibrosis. Transplantation 2012; 93:1136-1146.

29. Banon-Maneus E, Diekmann F, Carrascal M, et al. Two-dimensional difference in gel electrophoresis urinary proteomic profile in the search of nonimmune chronic allograft dysfunction biomarkers. Transplantation 2010; 89:548-558.

30. Quintana LF, Sole-Gonzalez A, Kalko SG, et al. Urine proteomics to detect biomarkers for chronic allograft dysfunction. J Am Soc Nephrol 2009; 20:428-435.

31. Quintana LF, Campistol JM, Alcolea MP, et al. Application of label-free quantitative peptidomics for the identification of urinary biomarkers of kidney chronic allograft dysfunction. Mol Cell Proteomics 2009; 8:1658-1673.

32. Ho J, Kreidberg JA. The long and short of microRNAs in the kidney. J Am Soc Nephrol 2012; 23:400-404.

33. Maluf DG, Dumur $\mathrm{Cl}$, Suh JL, et al. The urine microRNA profile may help monitor posttransplant renal graft function. Kidney Int 2014; 85:439-449.

34. Scian MJ, Maluf DG, David KG, et al. MicroRNA profiles in allograft tissues and paired urines associate with chronic allograft dysfunction with IF/TA. Am J Transplant 2011; 11:2110-2122.

35. Nauta FL, Bakker SJ, van Oeveren W, et al. Albuminuria, proteinuria, and novel urine biomarkers as predictors of long-term allograft outcomes in kidney transplant recipients. Am J Kidney Dis 2011; 57:733-743.

36. Szeto CC, Kwan BC, Lai KB, et al. Urinary expression of kidney injury markers in renal transplant recipients. Clin J Am Soc Nephrol 2010; 5:2329-2337.

37. Hall IE, Doshi MD, Reese PP, et al. Association between peritransplant kidney injury biomarkers and 1-year allograft outcomes. Clin J Am Soc Nephrol 2012; $7: 1224-1233$

38. Amer $\mathrm{H}$, Lieske JC, Rule AD, et al. Urine high and low molecular weight proteins one-year postkidney transplant: relationship to histology and graft survival. Am J Transplant 2013; 13:676-684.

39. Carr MW, Roth SJ, Luther E, et al. Monocyte chemoattractant protein 1 acts as a T-lymphocyte chemoattractant. Proc Natl Acad Sci U S A 1994; 91:3652-3656

40. Allavena $P$, Bianchi $G$, Zhou D, et al. Induction of natural killer cell migration by monocyte chemotactic protein-1, -2 and -3. Eur J Immunol 1994; 24:32333236.

41. Wang $\mathrm{T}$, Dai $\mathrm{H}, \mathrm{W}$ an $\mathrm{N}$, et al. The role for monocyte chemoattractant protein-1 in the generation and function of memory CD8+ T cells. J Immunol 2008; 180:2886-2893.

42. Ho J, Rush DN, Gibson IW, et al. Early urinary CCL2 is associated with the later development of interstitial fibrosis and tubular atrophy in renal allografts. Transplantation 2010; 90:394-400.
43. Ho J, Wiebe C, Gibson IW, et al. Elevated urinary CCL2: Cr at 6 months -1. is associated with renal allograft interstitial fibrosis and inflammation at 24 months. Transplantation 2014; 98:39-46.

This multicentre, prospective observational cohort study demonstrates that early urinary CCL2:Cr at 6 months predicts the subsequent development of IFTA and inflammation at 24 months, which is a significant predictor for late graft loss.

44. Ho J, Wiebe C, Rush DN, et al. Increased urinary CCL2: Cr ratio at 6 months is associated with late renal allograft loss. Transplantation 2013; 95:595602.

45. Qin S, Rottman JB, Myers $P$, et al. The chemokine receptors CXCR3 and CCR5 mark subsets of T cells associated with certain inflammatory reactions. J Clin Invest 1998; 101:746-754.

46. el-Sawy T, Fahmy NM, Fairchild RL. Chemokines: directing leukocyte infiltration into allografts. Curr Opin Immunol 2002; 14:562-568.

47. Dufour JH, Dziejman M, Liu MT, et al. IFN-gamma-inducible protein 10 (IP-10; CXCL10)-deficient mice reveal a role for IP-10 in effector T cell generation and trafficking. J Immunol 2002; 168:3195-3204.

48. Hricik DE, Nickerson $P$, Formica RN, et al. Multicenter validation of urinary CXCL9 as a risk-stratifying biomarker for kidney transplant injury. Am J Transplant 2013; 13:2634-2644.

49. Ho J, Wiebe C, Gibson IW, et al. Immune monitoring of kidney allografts. In translation series. Am J Kidney Dis 2012; 60:629-640.

50. Hirt-Minkowski $P$, Amico $P, H o J$, et al. Detection of clinical and subclinical tubulo-interstitial inflammation by the urinary CXCL10 chemokine in a real-life setting. Am J Transplant 2012; 12:1811-1823.

51. Schaub $S$, Nickerson $P$, Rush $D$, et al. Urinary CXCL9 and CXCL10 levels correlate with the extent of subclinical tubulitis. Am J Transplant 2009; 9:1347-1353.

52. Ho J, Rush DN, Karpinski M, et al. Validation of urinary CXCL10 as a marker of borderline, subclinical and clinical tubulitis. Transplantation 2011; 92:878882.

53. Blydt-Hansen TD, Gibson IW, Gao A, et al. Elevated urinary CXCL10-tocreatinine ratio is associated with subclinical and clinical rejection in pediatric renal transplantation. Transplantation 2015; 99:797-804.

54. Tatapudi RR, Muthukumar $T$, Dadhania $D$, et al. Noninvasive detection of renal allograft inflammation by measurements of mRNA for IP-10 and CXCR3 in urine. Kidney Int 2004; 65:2390-2397.

55. $\mathrm{Hu} \mathrm{H}$, Aizenstein BD, Puchalski $A$, et al. Elevation of CXCR3-binding chemokines in urine indicates acute renal-allograft dysfunction. Am J Transplant $2004 ; 4: 432-437$.

56. Hauser IA, Spiegler S, Kiss E, et al. Prediction of acute renal allograft rejection by urinary monokine induced by IFN- $\gamma$ (MIG). J Am Soc Nephrol 2005; 16:1849-1858.

57. Jackson JA, Kim EJ, Begley B, et al. Urinary chemokines CXCL9 and CXCL10 are noninvasive markers of renal allograft rejection and BK viral infection. Am J Transplant 2011; 11:2228-2234.

58. Peng $\mathrm{W}$, Chen J, Jiang $\mathrm{Y}$, et al. Urinary fractalkine is a marker of acute rejection. Kidney Int 2008; 74:1454-1460.

59. Matz M, Beyer J, Wunsch D, et al. Early posttransplant urinary IP-10 expression after kidney transplantation is predictive of short- and long-term graft function. Kidney Int 2006; 69:1683-1690. 